\title{
Produção de bebida fermentada enriquecida com ora-pro-nóbis (Pereskia aculeata)
}

André Vinícius Pocai, João Borges Laurindo, Maximiliano Segundo Escalona Jiménez, Neila Silvia Pereira dos Santos Richards

https://doi.org/10.4322/mp.978-65-991393-2-1.c4

\section{Resumo}

$\mathrm{O}$ aumento na procura por alimentos com propriedades funcionais leva à pesquisa e ao desenvolvimento de novos produtos constantemente. O leite fermentado é um alimento comum e que está presente na alimentação de pessoas de todo o mundo há séculos, porém nota-se que há no mercado cada vez mais tipos deste produto, seja com propriedades probióticas ou leites fermentados enriquecidos com algum componente, sejam vitaminas, fibras ou proteínas. Hortaliças não convencionais como a ora-pro-nóbis são uma alternativa alimentar no enriquecimento de produtos com proteínas de origem vegetal. A ora-pro-nóbis já está amplamente difundida em algumas regiões do Brasil como, por exemplo, Minas Gerais, Goiás, Espírito Santo, São Paulo, Rio de Janeiro, Paraná, Santa Catarina, Maranhão, Pernambuco, Bahia, Alagoas e Sergipe, sendo considerada um complemento nutricional devido ao seu conteúdo proteico que pode atingir, nas folhas 25\%, possuindo ainda fibras, ferro, cálcio, entre outros. A adição dessa cactácea torna-se uma interessante alternativa na elaboração de um produto, como o iogurte, com alto teor proteico, pois aumenta o teor das proteínas presentes no leite, resultando em um leite fermentado enriquecido. Este estudo objetivou a elaboração de quatro iogurtes com adição de ora-pro-nóbis. Um delineamento fatorial $2 \times 2$ foi utilizado e as formulações foram caracterizadas, físicoquímica, microbiológica e sensorialmente. Os iogurtes desenvolvidos apresentaram teor de proteína de $14 \%$ a $100 \%$ quando comparado com o produto sem adição de ora-pro-nóbis. Os produtos desenvolvidos atenderam a legislação vigente quanto número de microrganismos probióticos, sendo, também, aceitos sensorialmente pelos consumidores.

Palavras-chave: Análise sensorial, cactácea, delineamento fatorial, funcional, iogurte, probióticos.

\section{Introdução}

Preferencias alimentares desempenham um papel fundamental no consumo de derivados lácteos, principalmente por consumidores ávidos a novidades. Neste contexto, uma das preocupações da indústria de laticínios é a de atender a essa demanda colocando no mercado productos diferenciados e com maior valor agregado. Os productos demandados devem ser saudáveis, atrativos e sustentáveis, possuindo uma estreita relação com as necesidades e tendencias, ou mesmo, modas de consumo, fazendo com que indústria se adapte e apresente respostas rápidas às mudanças do mercado consumidor [1]. 
A fermentação é um dos métodos mais antigos utilizados para transformar leite em um produto com maior vida útil [2]. Não há evidencias precisas que indiquem a origem exata do iogurte, mas sabe-se que seus benefícios nutricionais e da saúde existiram em diversas civilizações ao longo dos anos [3]. O iogurte é um dos alimentos mais comuns existentes no mundo, consumido em diversos locais e com inúmeras variações. Seu consumo vai além apenas dos benefícios nutricionais, pois é benéfico para a saúde, como por exemplo, para a microflora intestinal, entre outros [4]. Por mais que seja feito há diversos séculos, o iogurte passa por inúmeras pesquisas de fabricação e rendimento, principalmente para aumentar a produção e garantir a segurança e qualidade do produto ao consumidor. Foram tomadas medidas como seleção dos microrganismos adequados à produção, tempo de fermentação e temperatura, entre outros pontos [3].

A Ora-pro-nóbis (Pereskia aculeata), é uma planta da família das cactáceas, com folhas desenvolvidas e originária das Américas, sendo encontrada do Brasil até a Flórida. Tornou-se uma importante fonte de alimento por não apresentar toxicidade e ter um alto teor de proteínas, fibras e outros nutrientes [5, 6]. Rocha et al. [7] sugerem que, por apresentar alto teor proteico em suas folhas, a ora-pronóbis pode ser denominada "carne dos pobres", mesmo que as proteínas de origem vegetal sejam consideradas de baixo valor biológico, pois são incompletas quanto à composição dos aminoácidos. A ora-pro-nóbis é uma planta alimentícia não convencional que se destaca das demais, pois possui substâncias bioativas, que a torna um alimento com propriedades funcionais [8]. Pelas suas características, essa planta representa uma alternativa para o enriquecimento de alimentos $[9,10]$.

Diante do exposto, neste trabalho foi feito o estudo da adição de ora-pro-nóbis desidratada ao iogurte, com o intuito de enriquecê-lo, tornando-o um alimento com alto teor proteico.

\section{Materiais e Métodos}

Foram preparadas duas amostras com leite UHT (Santa Clara ${ }^{\circledR}$ ): A1 contendo 8\% de açúcar e A2 com 10\% de açúcar (União ${ }^{\circledR}$ ) (Tabela1). Posteriormente, foram aquecidas a $83^{\circ} \mathrm{C}$ por 20 minutos para a pasteurização e completa dissolução do açúcar, sendo, então, resfriadas a $41 \pm 1{ }^{\circ} \mathrm{C}$. Após, adicionou-se assepticamente $1 \%$ do fermento lácteo BioRich (CHR Hansen $\left.{ }^{\circledR}\right)$, composto por culturas de Lactobacillus acidophilus

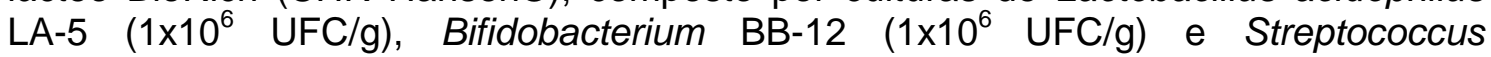
thermophilus. Com o auxílio de um bastão de vidro esterilizado as amostras A1 e A2 foram homogeneizadas, colocadas em frascos pirex esterilizados e levadas ao banho de água termostatizado $\left(42 \pm 1^{\circ} \mathrm{C}\right)$. Uma amostra controle (N1) foi também elaborada (Tabela 1). O processo de fermentação das amostras foi interrompido quando o pH atingiu o valor de 4,5, aproximadamente quatro horas. Após, foram resfriadas a temperatura ambiente $\left(20^{\circ} \mathrm{C}\right)$ e colocadas a $8^{\circ} \mathrm{C}$. As amostras A1 e A2 foram divididas em alíquotas e elaboradas as quatro formulações (N2 a N5) utilizando planejamento experimental fatorial 2X2 (Tabela 1). Em todas as formulações foi adicionado $1 \%$ de aroma sabor morango.

As amostras de ora-pronóbis foram obtidas já desidratadas e trituradas, provenientes de Porto Belo - SC, vendidas pelo Sítio Flora Bioativas. O aroma utilizado no estudo foi sabor morango (Duas Rodas ${ }^{\circledR}$ ).

Os parâmetros físico-químicos determinados nas formulações foram teores de: proteína por micro-Kjeldahl, gordura por butirômetro, umidade, cinzas, e carboidratos obtidos por diferença, sendo todas as análises realizadas em triplicatas. Os métodos 
seguiram as recomendações da Instrução Normativa no 68 de 2006 e/ou do Instituto Adolfo Lutz [11, 12]. Para a quantificação de Lactobacillus, utilizou-se o Agar MRS acidificado, com incubação a $37 \pm 1{ }^{\circ} \mathrm{C}$ durante 72 horas sob condições de anaerobiose. Os microrganismos do gênero Bifidobacterium foram quantificados em ágar MRS suplementado com cloreto de lítio $(0,1 \%)$, cisteína- $\mathrm{HCl}(0,05 \%)$ e dicloxacilina $(0,5 \mathrm{mg} / \mathrm{L})$, com incubação a $37 \pm 1^{\circ} \mathrm{C}$ por 72 horas em anaerobiose [13].

\begin{tabular}{c|c|c|c}
\hline Formulações & \% de ora-pro-nóbis & \% açúcar & \% aroma \\
\hline N1 & -- & -- & -- \\
N2 & 0,4 & 8 & 1 \\
N3 & 0,2 & 8 & 1 \\
N4 & 0,4 & 10 & 1 \\
N5 & 0,2 & 10 & 1 \\
\hline
\end{tabular}

Tabela 1. Formulações de iogurte utilizando o planejamento fatorial 2x2.

Os resultados físico-químicos e sensoriais foram analisados estatisticamente pela análise de variância e comparação das médias de pares de amostras pelo teste de Tukey ao nível de significância de 5\% [14, 15]. Para assegurar a qualidade dos iogurtes produzidos foram realizadas análises de coliformes termotolerantes e Salmonella, conforme a Resolução RDC $n^{\circ}$ 12, de 02 de janeiro de 2001 [16]. Para validação sensorial, foram feitos três testes [17] com 50 provadores, (estudantes e servidores) do departamento de Engenharia de Alimentos e Engenharia Química (CTC - EQA) da Universidade Federal de Santa Catarina (UFSC). O primeiro teste foi a escala hedônica, na qual o avaliador após provar uma amostra de cada vez, individualmente, atribui uma nota de acordo com sua preferência, sendo de 1 (desgostei muito) a 9 (gostei muitíssimo). O segundo foi o teste de ordenação, onde o avaliador coloca em ordem decrescente as amostras, de acordo com a sua preferência ( $1^{\circ}$ o que mais gostou e em $4^{\circ}$ o que menos gostou). O terceiro, de intenção de compra, onde o avaliador indica se compraria iogurte adicionado de ora-pro-nóbis, respondendo sim ou não. O teste de aceitação foi analisado pelo índice de aceitabilidade de cada amostra; já para o teste de ordenação-preferência, pelo método analítico de Friedman [17] e o teste de intenção de compra através de análise de frequência (\%).Para o cálculo do Índice de Aceitabilidade (I.A) de cada preparação, foi utilizada a seguinte expressão [18]:

$\mathrm{I} A(\%)=\mathrm{A} \times 100 / \mathrm{B}$

Em que:

A = nota média obtida para o produto;

$\mathrm{B}=$ nota máxima dada ao produto

\section{Resultados e Discussão}

A Tabela 2 mostra os resultados físico-químicos das formulações de iogurte desenvolvidas. Tendo em vista que a ora-pro-nóbis é uma cactácea rica em proteínas $[10,9]$, é esperado que a concentração proteica do iogurte aumente, conforme observado na Tabela 2.

Como esperado, as amostras contendo ora-pro-nóbis apresentaram a maior concentração proteica, em comparação à amostra controle, tendo em vista que este foi o motivo de suplementar o iogurte com esta planta. Dentre elas, a amostra N2, que contém $0,4 \%$ de ora-pro-nóbis e $8 \%$ de açúcar, foi a que apresentou maior 
concentração, em torno de $5,46 \%$, seguida da amostra N4, que contém $0,4 \%$ de orapro-nóbis e $10 \%$ de açúcar, com $4,58 \%$ de proteínas. As demais amostras, N3 e N5, por conterem apenas $0,2 \%$ de ora-pro-nóbis em sua composição, apresentaram menores concentrações de proteínas, 3,71\% e 3,09\% respectivamente.

\begin{tabular}{lccccc}
\hline $\begin{array}{c}\text { Análises/ } \\
\text { Formulações }\end{array}$ & $\begin{array}{c}\text { Proteína } \\
\left(\mathrm{g} \cdot 100 \mathrm{~g}^{-1}\right)\end{array}$ & $\begin{array}{c}\text { Gordura } \\
\left(\mathrm{g} \cdot 100 \mathrm{~g}^{-1}\right)\end{array}$ & $\begin{array}{c}\text { Umidade } \\
\left(\mathrm{g} \cdot 100 \mathrm{~g}^{-1}\right)\end{array}$ & $\begin{array}{c}\text { Cinzas } \\
\left(\mathrm{g} \cdot 100 \mathrm{~g}^{-1}\right)\end{array}$ & $\begin{array}{c}\text { Carboidratos } \\
\left(\mathrm{g} \cdot 100 \mathrm{~g}^{-1}\right)\end{array}$ \\
\hline N1 & $2,72 \pm 0,02^{\mathrm{e}}$ & $2,38 \pm 0,01^{\mathrm{c}}$ & $88,96 \pm 0,01^{\mathrm{a}}$ & $0,78 \pm 0,01^{\mathrm{a}}$ & $2,42 \pm 0,01^{\mathrm{e}}$ \\
\hline N2 & $5,46 \pm 0,01^{\mathrm{a}}$ & $2,58 \pm 0,01^{\mathrm{a}}$ & $81,69 \pm 0,01^{\mathrm{b}}$ & $0,70 \pm 0,01^{\mathrm{b}}$ & $12,31 \pm 0,01^{\mathrm{c}}$ \\
\hline N3 & $3,71 \pm 0,01^{\mathrm{c}}$ & $2,45 \pm 0,01^{\mathrm{b}}$ & $81,33 \pm 0,02^{\mathrm{c}}$ & $0,67 \pm 0,01^{\mathrm{c}}$ & $11,84 \pm 0,01^{\mathrm{d}}$ \\
\hline N4 & $4,58 \pm 0,01^{\mathrm{b}}$ & $2,24 \pm 0,02^{\mathrm{d}}$ & $79,97 \pm 0,03^{\mathrm{d}}$ & $0,71 \pm 0,01^{\mathrm{b}}$ & $12,51 \pm 0,01^{\mathrm{b}}$ \\
\hline N5 & $3,09 \pm 0,02^{\mathrm{d}}$ & $2,40 \pm 0,01^{\mathrm{c}}$ & $79,94 \pm 0,01^{\mathrm{d}}$ & $0,69 \pm 0,01^{\mathrm{bc}}$ & $13,87 \pm 0,01^{\mathrm{a}}$ \\
\hline
\end{tabular}

Legenda: letras iguais na mesma coluna indicam que não houve diferença estatística significativa a $5 \%(\mathbb{2} \leqslant 0,05)$. N1: iogurte natural; N2: iogurte com adição de $0,4 \%$ de ora -pronóbis, $8 \%$ de açúcar e 1\% de aroma; N3: iogurte com adição de 0,2\% de ora-pro-nóbis, $8 \%$ de açúcar e 1\% de aroma; N4: iogurte com adição de 0,4\% de ora-pro-nóbis, $10 \%$ de açúcar e 1\% de aroma; N5: iogurte com adição de $0,2 \%$ de ora-pro-nóbis, $10 \%$ de açúcar e $1 \%$ de aroma Tabela 2. Composição centesimal das formulações de iogurte adicionado de ora-pronóbis.

A Organização Mundial da Saúde (WHO) [19] determinou que um consumo saudável de proteínas ficasse em torno de 0,83 g kg-1 por dia, para adultos de ambos os sexos, o que para uma pessoa de $60 \mathrm{~kg}$, seriam aproximadamente $50 \mathrm{~g} \mathrm{dia}^{-1}$. Com consumo de iogurte enriquecido com $0,4 \%$ ora-pro-nóbis, as necessidades diárias seriam supridas em aproximadamente $10 \%$ em apenas $100 \mathrm{~g}$ de produto, mostrando-se uma excelente fonte proteica para pessoas que buscam uma dieta saudável. Para crianças e adolescentes, a WHO determina que há uma faixa mais restrita no consumo de proteínas, variando de acordo com a idade, peso e sexo. Para meninos de sete a dez anos, por exemplo, o consumo médio ficaria em torno de $25,9 \mathrm{~g} \mathrm{dia}^{-1}$, o que seria suprido em $20 \%$ com o consumo de $100 \mathrm{~g}$ de iogurte enriquecido (N2).

Segundo Rocha et al. [6], a ora-pro-nóbis é uma planta que possui, quando desidratada, em torno de $3,64 \%$ de lipídios em sua composição, o que a torna um alimento que pode ser utilizado em dietas com restrições de gordura. A adição desta planta no iogurte não caracterizou variações da quantidade de gordura em comparação à amostra controle.

Com o resultado das análises, nota-se que os valores de concentração de gordura nas amostras com ora-pro-nóbis variaram de 2,24 a 2,58 g.100 g $\mathrm{g}^{-1}$, sendo que a amostra N5 não diferiu significativamente da amostra controle, porém ambas diferiram das demais amostras. Conforme pode ser observado na Tabela 2 a adição de ora-pronóbis, não contribuiu para o incremento do teor de gordura das amostras, indicando que a planta realmente possui baixo teor de lipídios e não interfere neste quesito nos productos elaborados.

Por se tratar de um alimento rico em fibras [5, 20], era esperado que a umidade do alimento tendesse a aumentar, tendo em vista que as fibras tendem a aprisionar mais umidade, porém notou-se que a umidade diminui principalmente nas formulações N4 e N5 e não apresentaram diferenças significativas entre si, provavelmente devido a presença da maior concentração de açúcar (10\%), aumentando o efeito osmótico da amostra, diminuindo a umidade, como pode ser visto na Tabela 2. A amostra controle (N1) foi a que apresentou o maior teor de umidade, diferindo significativamente das demais amostras. 
O teor de minerais no corpo humano desempenha um papel importante quanto ao bom funcionamento do organismo, tais como o metabolismo e constituição de tecidos [10]. A ora-pro-nóbis, segundo Takeiti et al. [7], apresenta grandes quantidades de vitaminas e minerais. Almeida et al. [21], Rodrigues [22] e Oliveira [23] destacaram que, entre outras hortaliças não convencionais, a ora-pro-nóbis se sobressaía principalmente pelas concentrações de magnésio, ferro, cálcio e manganês.

A amostra controle apresentou o maior teor de cinzas, diferindo das demais formulações. As amostras N2, N4 e N5 não diferiram entre si, já a amostra N3 não diferiu da amostra N5, porém, diferiu das demais formulações.

A amostra controle (N1) apresentou uma baixa concentração de carboidratos em sua composição, 2,42\% em média. Conforme mostrado na Tabela 2, com a adição de açúcar nas formulações, o teor de carboidratos variou de 11,84 a $13,87 \mathrm{~g} .100 \mathrm{~g}^{-1}$. Apesar da adição ter sido entre 8 e $10 \%$ de açúcar, a ora-pro-nóbis apresenta em sua composição em torno de $36,2 \%$ de carboidratos [6].

$\mathrm{O}$ pH de um iogurte comum é em torno de 4,6 [24], isso garante a correta coagulação do leite, pois este é o ponto isoelétrico da caseína. As amostras estudadas apresentaram pH um pouco abaixo de 4,6, entre 4,17 e 4,34, porém isso não indicou nenhuma perda nas características físicas do iogurte. A Tabela 3 apresenta os valores de $\mathrm{pH}$ obtidos nas formulações e na amostra controle.

\begin{tabular}{cc}
\hline Formulações & Valor de $\mathrm{pH}$ \\
\hline N1 & $4,21 \pm 0,01^{\mathrm{a}}$ \\
N2 & $4,17 \pm 0,02^{\mathrm{a}}$ \\
N3 & $4,23 \pm 0,02^{\mathrm{a}}$ \\
N4 & $4,34 \pm 0,02^{\mathrm{a}}$ \\
N5 & $4,25 \pm 0,02^{\mathrm{a}}$ \\
\hline
\end{tabular}

Tabela 3. Valor de $\mathrm{pH}$ das formulações de iogurte desenvolvidas com adição de orapro-nóbis

Os valores encontrados indicam que a adição de açúcar e ora-pro-nóbis não interferiram na acidificação do meio, tendo em vista que todos flutuaram em torno do $\mathrm{pH}$ da amostra controle, 4,21.

A RDC $n^{0}$ 12, de 02 de janeiro de 2001 [16], estabelece o Regulamento Técnico sobre Padrões Microbiológicos para Alimentos, o qual possui em seu anexo valores máximos para a análise de coliformes termotolerante, no caso seriam 10 NMP/g, e Salmonella, que tem como valor ausência em $25 \mathrm{~g}$.

Em ambas as análises, para as cinco amostras (N1, N2, N3, N4 e N5), obteve-se valores aceitáveis perante a legislação. Para Salmonella, obteve-se a ausência em 25 $\mathrm{g}$ para as cinco amostras, e para a análise de coliformes termotolerantes nas três diluições requeridas, $10^{-1}, 10^{-2}$ e $10^{-3}$, não observou-se tubos positivos, indicando que o número mais provável é menor que três por grama de amostra.

A Tabela 4 mostra os resultados das análises para os iogurtes N1 a N5, quanto à quantidade de Lactobacillus e Bifidobacterium.

De acordo com a legislação brasileira para alimentos probióticos a quantidade mínima viável deve estar situada na faixa de $10^{8}(8 \mathrm{log})$ a $10^{9}(9 \mathrm{log})$ unidades formadoras de colônias (UFC. $\mathrm{g}^{-1}$ ou $\mathrm{mL}^{-1}$ ) na recomendação diária do produto pronto para $\mathrm{o}$ consumo, valores menores podem ser aceitos, desde que comprovada sua eficacia. 
Dessa forma todos os iogurtes produzidos neste estudo apresentaram contagem dentro do preconizado pela legislação brasileira [25].

\begin{tabular}{ccc}
\hline Formulações & $\begin{array}{c}\text { Lactobacillus LA-5 } \\
\text { Log UFC. } .^{-1}\end{array}$ & $\begin{array}{c}\text { Bifidobacterium BB-12 } \\
\text { Log UFC. } \text { g }^{-1}\end{array}$ \\
\hline N1 & 8,31 & 8,09 \\
N2 & 8,17 & 7,61 \\
N3 & 8,69 & 8,12 \\
N4 & 8,35 & 7,95 \\
N5 & 8,75 & 8,24 \\
\hline
\end{tabular}

Tabela 4. Logaritmos das médias do número de unidades formadoras de colônias $\left(\right.$ UFC. ${ }^{-1}$ ) de Lactobacillus e Bifidobacterium observadas nos iogurtes adicionados de ora-pro-nóbis.

Os probióticos são definidos internacionalmente como microrganismos vivos que quando administrados em quantidade adequadas, conferem beneficios a saúde do hospedeiro. Os beneficios dos probióticos sobre o microbioma humano inclui fatores como efeitos antagônicos, competição e efeitos imunológicos, resultando no aumento da resistência contra patógenos. Portanto, com a utilização de culturas bacterianas probióticas há um estímulo da multiplicação de bactérias benéficas, em detrimento da proliferação de bactérias potencialmente prejudiciais, reforçando os mecanismos naturais de defesa do hospedeiro $[1,26]$.

Os lactobacilos são reconhecidos por sua atuação na promoção de saúde e manutenção equilibrada da microbiota intestinal na qual, pode diminuir os procesos putrefativos por reduzir o acúmulo de substâncias nocivas no trato gastrointestinal. A presença de níveis elevados de bacterias bífidas está relacionado com uma microbiota intestinal saudável em todas as fases de vida da espécie humana [27, 28].

Alguns fatores podem afetar o crescimento e a sobrevivência dos microrganismos probióticos, como, por exemplo, valor de $\mathrm{pH}$, teor de gordura, a concentração e tipo de proteínas, açúcares, minerais e vitaminas [29, 30]. No desenvolvimento de novos produtos com alegação probiótica, esses devem fazer parte da dieta normal do indivíduo, para que seja mantido o nível terapéutico mínimo diario recomendável. As formulações N3 e N5 que continham $0,2 \%$ de ora-pro-nóbis apresentam uma contagem superior quando comparada com as demais formulações, independente da concentração de açúcar presente no produto, sugerindo que esta concentração de ora-pro-nóbis tenha, provavelmente, colaborado para o melhor desenvolvimento das bacterias probióticas presentes nos iogurtes.

$\mathrm{Na}$ análise sensorial o ser humano é um importante instrumento de medida das características sensoriais dos alimentos. Na Tabela 5 estão expressadas as médias do teste de aceitação e o índice de aceitabilidade dos iogurtes adicionados de ora-pronóbis e açúcar.

\begin{tabular}{c|c|c}
\hline Formulações & Média & $\begin{array}{c}\text { Índice de aceitabilidade } \\
\text { (\%) }\end{array}$ \\
\hline N2 & $6,74 \pm 0,82$ & 74,89 \\
N3 & $7,78 \pm 0,38$ & 86,44 \\
N4 & $6,98 \pm 0,80$ & 77,56 \\
N5 & $7,66 \pm 0,43$ & 85,11 \\
\hline
\end{tabular}

Tabela 5. Resultado (média \pm desvio-padrão, $n=50$ ) das notas atribuídas pelos julgadores e índice de aceitabilidade das formulações de iogurte adicionado de orapro-nóbis. 
No desenvolvimento de um novo produto, um dos pontos principais é avaliar sua aceitabilidade, com o intuito de predizer sua aceitação frente ao mercado consumidor [31]. Segundo Teixeira et al. [32] e Dutcosky [17], para que o produto seja considerado como aceito, em termos de suas propriedades sensoriais, é necessário que obtenha um Î́ndice de Aceitabilidade (IA) de, no mínimo, 70\%. Com base nas médias obtidas no teste de aceitação e no cálculo do IA, verifica-se que todas as formulações de iogurte apresentaram boa aceitabilidade. As formulações N3 e N5 apresentaram IA superior a $80 \%$, indicando a tendencia de preferência pelos julgadores.

A Tabela 6 apresenta resultados do teste de ordenação-preferência, observa-se que a amostra N2 difere das amostras N3, N4 e N5, sendo que as três últimas não apresentam diferenças entre si. A formulação N5 apresentou uma tendencia de preferencia, porém não diferiu das demais formulações (N3 e N4).

\begin{tabular}{l|c|c|c|c|c}
\hline \multirow{2}{*}{ Formulações } & \multirow{2}{*}{ Total } & \multicolumn{4}{|c}{ Amostras } \\
\cline { 3 - 6 } & & 159 & 115 & 124 & 102 \\
\cline { 3 - 6 } & 159 & -- & $44^{*}$ & $35^{*}$ & $57^{*}$ \\
\hline N2 & 115 & & -- & $9^{\text {ns }}$ & $13^{\text {ns }}$ \\
\hline N3 & 124 & & & -- & $22^{\text {ns }}$ \\
\hline N4 & 102 & & & & -- \\
\hline
\end{tabular}

*As amostras diferem entre si; ns = as amostras não diferem entre si a $p \leq 0,05$

N2 - 0,4\% de ora-pro-nóbis e $8 \%$ de açúcar; N3 - 0,2\% de ora-pro-nóbis e 8\% de açúcar; N4 $0,4 \%$ de ora-pro-nóbis e $10 \%$ de açúcar; N5 - 0,2\% de ora-pro-nóbis e 10\% de açúcar.

Tabela 6. Resultado do teste de ordenação-preferência das amostras de iogurte adicionados de ora-pro-nóbis.

Os resultados obtidos no teste de intenção de compra composta de cinco pontos estão apresentados na Tabela 7. Dos 50 participantes do teste sensorial, 48 indicaram que sim, comprariam um iogurte enriquecido com ora-pro-nóbis, sendo que apenas 2 indicaram que não o comprariam. Isso resulta em uma aceitabilidade de $96 \%$ dos participantes.

\section{Conclusão}

A adição de ora-pro-nóbis, mostrou-se uma alternativa interessante para o enriquecimento de iogurte, tendo em vista principalmente o aumento considerável de proteínas. As análises microbiológicas indicaram que a adição de ora-pro-nóbis não influenciou na qualidade e segurança do alimento, pois os resultados foram todos satisfatórios e de acordo com a legislação vigente. Os iogurtes desenvoltos podem ser considerados como probióticos, uma vez que a contagem de microrganismos está de acordo com a preconizada pela legislação. O índice de aceitação do iogurte também foi satisfatório, principalmente se for levado em conta que todas as quatro formulações foram aceitas pelos julgadores. Vale ressaltar que houve julgadores que gostaram do iogurte, porém acharam um pouco adocicados demais, ou sugeriram a utilização de adoçantes naturais na formulação, por exemplo, mas que no geral acharam a ideia de consumir um produto enriquecido com um produto natural uma alternativa viável.

\section{Referências}

[1] Richards N.S.P.S. Novos produtos para a indústria de laticínios. In: Martins P.C., Piccinini G.A., Krug E.E.B., Martins C.E., Lopes F.C.F. (eds) Sustentabilidade ambiental, social e econômica da cadeia produtiva do leite. Brasília: EMBRAPA, 2015, p. 329-338. Brasília, Brasil. 
[2] Pederson C.S. Microbiology of Food Fermentations. Starch, Connecticut 1979; 32(4):1-29.

[3] Tamime A.Y., Robinson R.K. Yoghurt: Science and Technology. Woodhead Publishing Limited, 2007. 808p.

[4] Buttrius J. Nutritional properties of fermented milk products. International Journal of Dairy Technology 1997; 50:21-27.

[5] Mercê A.L.R. Fernandes E., Mangrich A.S., Sierakowski M.R., Szpoganicz B. $\mathrm{Fe}(\mathrm{III})$ : galactomannan solid and aqueous complexes: potentiometric, EPR spectroscopy and thermal data. Journal of Brazilian Chemical Society, Campinas 2001; 12(6):791-798. https://doi.org/10.1590/S0103-50532001000600017.

[6] Rocha D.R.C., Pereira Junior G.A., Vieira G., Pantoja L., Santos A.S., Pinto N.A.V.D. Macarrão adicionado de ora-pro-nóbis (Pereskia aculeata Miller) desidratado. Alimentos e Nutrição, Araraquara 2008; 19(4):459-465.

[7] Takeiti C.Y., Antonio G.C., Motta E.M.P., Collares-Queiroz F.P., Park K.J. Nutritive evaluation of non-conventional leafy vegetable (Pereskia aculeata Miller). International Journal of Food Sciences and Nutrition, Hants 2009; 60(1):148-160. https://doi.org/10.1080/09637480802534509.

[8] Almeida M.E.F. Farinha de folhas de cactáceas do gênero Pereskia: caracterização nutricional e efeito sobre ratos wistar submetidos à dieta hipercalórica. Tese de Doutorado - Curso de Agroquímica, Universidade Federal de Lavras, Lavras, 2012.

[9] Mercê A.L.R., Landaluze J.S., Mangrich A.S., Szpoganicz B., Sierakowski M.R. Complexes of arabinogalactan of Pereskia aculeata and $\mathrm{Co}^{2+}, \mathrm{Cu}^{2+}, \mathrm{Mn}^{2+}$, and $\mathrm{Ni}^{2+}$. Bioresource Technology 2001; 76(1):29-37. https://doi.org/10.1016/S09608524(00)00078-X.

[10] Ribeiro A.S. Estudo de plantas alimentícias não convencionais e aplicação em productos lácteos. Tese de Doutorado. Santa Maria: Universidade Federal de Santa Maria; 2019.

[11] Brasil, Ministério da Agricultura do Abastecimento e da Reforma Agrária (2006). Instrução Normativa no 68 de 12/12/2006. Métodos Analíticos Oficiais Físico-químicos para controle de leite e produtos lácteos. Disponível em: https://www.diariodasleis.com.br/busca/exibelink.php?numlink=1-77-23-2006-12-1268. Acesso em 13 jun. 2016.

[12] IAL. Instituto Adolfo Lutz. Métodos físico químicos para análise de alimentos - $4^{\mathrm{a}}$ ed. Série Normas e Manuais Técnicos, Ministério da Saúde, ANVISA: Brasília, 2008,1020 p.

[13] APHA. American Public Health Association (2001). Compendium of methods for the microbiological examination of foods. 4th ed. Washington: APHA. 676 p.

[14] Althaus R.A., Canteri M.G., Giglioti E.A. Tecnologia da informação aplicada ao agronegócio e ciências ambientais: sistema para análise e separação de médias pelos métodos de Duncan, Tukey e Scott-Knott, Anais do X Encontro Anual de Iniciação Científica 2001. Parte 1, Ponta Grossa, 280-281. 
[15] Canteri M.G., Althaus R.A., Virgens Filho J.S., Giglioti E.A., Godoy C.V. SASMAgri: Sistema para análise e separação de médias em experimentos agrícolas pelos métodos de Scott-Knott, Tukey e Duncan. Revista Brasileira de Agrocomputação 2001;1(2):18-24.

[16] ANVISA - Agência Nacional de Vigilância Sanitária. Brasil. Resolução $n^{\circ} 12$, de 2 de jaeiro de 2001. Aprova o Regulamento técnico sobre padres microbiológicos para alimentos. Disponivel em: www.anvisa.gov.br. Acesso em 13 de ago. de 2018.

[17] Dutcosky S.D. Análise sensorial de alimentos. Curitiba: Champagnat, 2007.158p

[18] Palermo J.R. Análise sensorial: fundamentos e métodos. Rio de Janeiro: Editora Atheneu, 2015. 158p.

[19] Mahan L.K., Raymond J.L. Krause Alimentos, Nutrição e Dietoterapia. $14^{\mathrm{a} e d .}$ 2018, Elsevier. 1160p.

[20] Carvalho C.M., Luz I.S., Santos D.B., Azevedo D.O., Gomes Filho R.R., Valnir Júnior M. Cultivo Adensado de Ora-Pro-Nóbis Irrigado No Território Do Sisal Baiano. Revista Brasileira de Agricultura Irrigada - RBAI 2019; 13(6):3765-3772. https://doi.org/10.7127/rbai.v13n6001161.

[21]Almeida M.E.F., Junqueira A.M.B., Simão A.A., Corrêa A.D. Caracterização química das hortaliças não-convencionais conhecidas como ora-pro-nóbis. Bioscience Journal 2014; 30(1):431-439. Disponìvel em:

http://www.seer.ufu.br/index.php/biosciencejournal/article/view/17555. 20 ago. 2020.

[22] Rodrigues A.S. Atividade antioxidante e antimicrobiana de extratos de ora-pronóbis (Pereskia aculeata Mill.) e sua aplicação em mortadela. 2016, Dissertação. Universidade Federa de Santa Maria, Santa Maria, RS. Disponível em: http://search.ebscohost.com/login.aspx?direct=true \&db=cat00956a\&AN=ufsm.0008802 7\&lang=pt-br\&site=eds-live\&scope=site. Acesso em: 11 ago. 2020.

[23] Oliveira D.C.S., Wobeto C., Zanuzo M.R., Severgnini C. Composição mineral e teor de ácido ascórbico nas folhas de quatro espécies olerícolas não-convencionais. Horticultura Brasileira 2013; 31(3):472-475. https://doi.org/10.1590/S010205362013000300021.

[24] Lee W.J., Lucey J.A. Formation and Physical Properties of Yogurt. AsianAustralasian Journal of Animal Sciences 2010; 23(9):1127-1136. https://doi.org/10.5713/ajas.2010.r.05.

[25] Agência Nacional de Vigilância Sanitária, Brasil. IX - Lista de alegações de propriedade funcional aprovada. In: Alimentos com Alegações de Propriedades Funcionais e ou de Saúde, Novos Alimentos/Ingredientes, Substâncias Bioativas e Probióticos. 2008. Disponível em:

http://portal.anvisa.gov.br/wps/content/Anvisa+Portal/Anvisa/Inicio/Alimentos/Assunto $\mathrm{s}+\mathrm{de}+$ Interesse/Alimentos+Com+Alegacoes+de+Propriedades+Funcionais+e+ou+de +Saude/Alegacoes+de+propriedade+funcional+aprovadas>. Acesso em: 13 jun. 2016.

[26] Lopez-Garcia E., Leon-Muñoz L., Guallar-Castillon P., Rodríguez-Artalejo F. Habitual Yogurt Consumption and Health-Related Quality of Life: A Prospective Cohort Study. Journal of the Academy of Nutrition and Dietetics 2015; 31-39. https://doi.org/10.1016/j.jand.2014.05.013. 
[27] Tulk H.M.F., Blonski D.C., Murch L.A., Duncan A.M., Wright A.J. Daily consumption of a synbiotic yogurt decreases energy intake but does not improve gastrointestinal transit time: a double-blind, randomized, crossover study in healthy adults. Nutrition Journal 2013; 12(1):87-90. https://doi.org/10.1186/1475-2891-12-87

[28] Ferreira C.L.L.F. Grupo de bactérias lácticas e aplicação tecnológica de bactérias probióticas. In: Ferreira, C.L.L.F. Prebióticos e probióticos: atualização e prospecção. Rio de Janeiro: Rubio, 2018. p. 1-18.

[29] Oliveira M.N. Incorporando probióticos em alimentos. In: In gut ww trust. São Paulo: Sarvier, 2013. p.55-102.

[30] Hemarajata P., Versalovic J. Effects of probiotics on gut microbiota: mechanisms of intestinal immunomodulation and neuromodulation. Therapeutic Advances In Gastroenterology 2012; 6(1):39-51. https://doi.org/10.1177/1756283X12459294

[31] Moscatto J.A., Prudêncio-Ferreira S.H., Hauly, M.C.O. Farinha de yacon e inulina como ingredientes na formulação de bolo de chocolate. Ciência e Tecnologia de Alimentos 2004; 24(4):634-640. http://dx.doi.org/10.1590/S0101-20612004000400026.

[32] Teixeira E., Meinert E., Barbeta P.A. Análise sensorial dos alimentos. Florianópolis: UFSC, $1987.182 \mathrm{p}$.

\section{Autores}

André Vinícius Pocai ${ }^{1}$, João Borges Laurindo ${ }^{1}$, Maximiliano Segundo Escalona Jiménez ${ }^{2}$, Neila Silvia Pereira dos Santos Richards ${ }^{2, *}$

1. Departamento de Engenharia Química e Engenharia de Alimentos - EQA, Universidade Federal de Santa Catarina, Campus Reitor João David Ferreira Lima, s/n - Bairro Trindade, 88040-970, Florianópolis - SC, Brasil.

2. Departamento de Tecnologia e Ciência dos Alimentos, Universidade Federal de Santa Maria - DTCA/UFSM, Avenida Roraíma, 1000, Prédio 42, sala 3211, 97105900, Santa Maria - RS, Brasil.

* Autor para correspondência: neilarichardsprof@gmail.com 